\title{
INJÚRIA PELO FRIO NA QUALIDADE PÓS-COLHEITA DE MANGAS CV. PALMER ${ }^{1}$
}

\author{
ANA CAROLINA ALMEIDA MIGUEL², JOSÉ FERNANDO DURIGAN ${ }^{3}$, \\ CRISTIANE MARIA ASCARI MORGADO ${ }^{4}$, RAMON FELIPE DE OLIVEIRA GOMES 5
}

RESUMO - Este trabalho objetivou estabelecer os binômios temperatura $\mathrm{x}$ tempo, que podem ocasionar injúria pelo frio em mangas 'Palmer'. Frutos colhidos no estádio "de vez" foram cuidadosamente transportados para laboratório, onde foram selecionados, padronizados quanto à coloração, ao tamanho e à ausência de injúrias, e tratados com fungicida antes de serem armazenados a $2{ }^{\circ} \mathrm{C}, 5{ }^{\circ} \mathrm{C}$ e $12{ }^{\circ} \mathrm{C}$, por até 28 dias. Os frutos foram avaliados semanalmente quanto à ocorrência de podridões, danos pelo frio, atividade respiratória, coloração da casca e da polpa, massa fresca, firmeza da polpa, teores de sólidos solúveis (SS) e de acidez titulável (AT), e relação SS/AT. Os resultados indicaram que os sintomas de injúrias pelo frio foram exteriorizados após 7 dias do armazenamento refrigerado a $2{ }^{\circ} \mathrm{C}$ ou $5{ }^{\circ} \mathrm{C}$, com os frutos não diferindo quanto à gravidade dos danos. A presença dos danos não impediu o desenvolvimento da coloração característica da polpa, porém levou ao escurecimento da casca e afetou o amadurecimento normal dos frutos. Frutos armazenados a $12{ }^{\circ} \mathrm{C}$ não apresentaram sinais de danos pelo frio ou prejuízos aos seus processos metabólicos normais.

Termos para indexação: Mangifera indica; pós-colheita; refrigeração.

\section{CHILLING INJURY IN MANGOES CV. PALMER}

\begin{abstract}
This study aimed to establish the binomials temperature $v s$. time, which can cause chilling injury in mangoes 'Palmer'. Semi-ripe fruits were harvested and carefully transported to the laboratory where they were selected, standardized according to the color, size and absence of injuries and treated with fungicide before being stored at $2{ }^{\circ} \mathrm{C}, 5^{\circ} \mathrm{C}$ and $12{ }^{\circ} \mathrm{C}$ up to 28 days. The fruits were evaluated weekly for the occurrence of decay, chilling injury, respiratory activity, peel and pulp color, fresh weight mass, firmness, soluble solids (SS) and titratable acidity (TA) and ratio. The results showed that the symptoms of chilling injuries were exteriorized after 7 days of refrigerated storage at $2{ }^{\circ} \mathrm{C}$ or $5{ }^{\circ} \mathrm{C}$, and the fruits did not differ in the severity of the damage. The presence of damage did not prevent the development of the characteristic pulp color, although it has led to the darkening of the skin and has affected the normal ripening of fruits. Fruit stored at $12{ }^{\circ} \mathrm{C}$ showed no signs of cold damage, and no impairment to their normal metabolic processes.
\end{abstract} Index terms: Mangifera indica; postharvest; refrigeration.

\section{INTRODUÇÃO}

A manga é considerada uma importante fruta tropical, pois destaca-se pelo excelente sabor e aroma, exótica coloração e tem crescente aceitação no mercado externo (LIMA et al., 2006). O Brasil, apesar de ser um grande produtor de mangas, exporta um volume ainda pequeno, devido à sua curta vida útil (30 dias), o que dificulta o uso de transporte marítimo para distâncias com mais de 20 dias de trânsito (FAO, 2011).
O armazenamento refrigerado é uma ferramenta muito importante para o prolongamento da vida útil de frutos e hortaliças. Infelizmente, frutos tropicais, a exemplo da manga, são geralmente sensíveis à disfunção fisiológica denominada chilling injury ou dano pelo frio, quando expostas a temperaturas inferiores a $7-13{ }^{\circ} \mathrm{C}$, resultando em perdas quantitativas e qualitativas pós-colheita (WANG et al., 2008). O armazenamento destes frutos a baixas temperaturas exige o conhecimento de seus processos metabólicos na época da colheita, bem como de

\footnotetext{
${ }^{1}$ Trabalho Sinfruit 106 - Simpósio Internacional de Fruticultura - Avanços na Fruticultura (17 a 21 Outubro) ${ }^{2}$ Doutoranda em Agronomia Faculdade de Ciências Agrárias e Veterinárias - FCAV/UNESP; Jaboticabal.

E-mail: anaamiguel@yahoo.com.br.

${ }^{3}$ Prof $^{\circ}$. do Departamento de Tecnologia da FCAV/UNESP, Jaboticabal. E-mail: jfduri@fcav.unesp.br.

${ }^{4}$ Doutoranda em Agronomia Faculdade de Ciências Agrárias e Veterinárias - FCAV/UNESP; Jaboticabal.

E-mail: cristianemorgado4@yahoo.com.br.

${ }^{5}$ Eng. Agrônomo, FCAV/UNESP, Jaboticabal. E-mail: rmn_unesp@yahoo.com.br.
} 
suas respostas, quando armazenado sob temperaturas críticas ou sob condição de dano pelo frio (MUÑOZ et al., 2001).

Além da temperatura, o tempo de exposição é determinante no desenvolvimento de injúrias pelo frio. Os sintomas comumente reportados são o amadurecimento irregular, o incompleto desenvolvimento da cor e do sabor, o aumento da suscetibilidade a doenças, a descoloração da casca e, em muitos casos, o escurecimento da polpa (WANG et al., 2008). Esses sintomas são agravados com a transferência da fruta para temperaturas mais elevadas, o que é dependente da variedade e do estádio de maturação, assim como do tempo e da temperatura de exposição ao longo do armazenamento (PHAKAWATMONGKOL et al., 2004).

Este trabalho objetivou determinar o binômio tempo $\mathrm{x}$ temperatura de armazenamento capaz de causar injúria pelo frio em mangas 'Palmer' e seu efeito na qualidade dos frutos.

\section{MATERIAL E MÉTODOS}

Frutos de mangeira 'Palmer' foram colhidos no estádio "de vez", em pomar comercial localizado no município de Monte Alto-SP, e imediatamente transportados ao Laboratório de Tecnologia dos Produtos Agrícolas, do Departamento de Tecnologia da FCAV/UNESP, onde tiveram seus pedúnculos padronizados em 10-20 mm, sendo lavados com detergente neutro, enxaguados em água corrente e novamente selecionados, eliminando-se os danificados ou desuniformes. Em seguida, os frutos foram tratados por imersão em fungicida Magnate $500 \mathrm{EC}^{\circledR}$ (Imazalil a $50 \%$ ) a $200 \mathrm{~mL} 100 \mathrm{~L}^{-1}$, a $10^{\circ} \mathrm{C}$ por $2 \mathrm{~min}$, e armazenados a $2 \pm 0,4^{\circ} \mathrm{C}(75,7 \pm 15,6 \%$ UR $), 5 \pm 1^{\circ} \mathrm{C}$ $\left(73,8 \pm 12,8 \%\right.$ UR) e $12 \pm 0,9{ }^{\circ} \mathrm{C}(82 \pm 9,7 \%$ UR $)$, com avaliações realizadas aos $0 ; 7 ; 14 ; 21$ e 28 dias.

Os frutos foram avaliados quanto à incidência de podridões, o que foi realizado visualmente, contando-se o número de mangas com presença de podridões, cujas lesões apresentavam diâmetro superior a $0,5 \mathrm{~cm}$. Os danos pelo frio foram estimados através de observação visual e atribuição de notas: $1=$ severo ( $>50 \%$ da superfície dos frutos injuriada); $2=$ moderado (25-50\% da superfície injuriada pelo frio); $3=$ brando/suave (até $25 \%$ da superfície contendo depressões e escaldadura); $4=$ leve $(2-5 \%$ da superfície danificada); $5=$ sem sintomas visíveis de injúria (WHANGCHAI et al., 2000). A atividade respiratória foi determinada mantendo-se dois frutos em recipiente hermeticamente fechado com capacidade de $3 \mathrm{~L}$, por uma hora. Através de septo de silicone, coletaram-se alíquotas de $0,3 \mathrm{~mL}$ da atmosfera interna do recipiente, antes e após o fechamento, a qual foi analisada em cromatógrafo a gás GC Finnigan 9001. Os resultados foram expressos em $\mathrm{mL}$ de $\mathrm{CO}_{2} \mathrm{~kg}^{-1} \mathrm{~h}^{-1}$. A perda de massa fresca foi determinada por pesagem em balança Marte mod. AS 2000, e a coloração da casca e da polpa utilizando-se colorímetro Minolta CR 400b, com os resultados expressos em luminosidade, ângulo hue e cromaticidade. A firmeza da polpa foi determinada, utilizando-se penetrômetro, aplicado em dois pontos opostos na região equatorial do fruto, com os resultados expressos em Newton $(\mathrm{N})$.

$\mathrm{Na}$ polpa dos frutos, foram determinados os teores de sólidos solúveis (SS) em refratômetro digital e acidez titulável (AT), de acordo com a metodologia descrita pela AOAC (2005), o que permitiu calcular a relação SS/AT.

O delineamento experimental adotado foi o inteiramente casualizado, disposto em esquema fatorial $3 \times 5$, com três repetições constituídas de dois frutos cada, em que o primeiro fator correspondeu às temperaturas $\left(2^{\circ} \mathrm{C}, 5^{\circ} \mathrm{C}, 12^{\circ} \mathrm{C}\right)$ e o segundo, ao tempo de armazenamento $(0 ; 7 ; 14 ; 21$ e 28 dias $)$. Os dados obtidos foram submetidos à análise de variância, pelo teste $\mathrm{F}$, e comparação das médias pelo teste de Tukey, a 5\% de probabilidade.

\section{RESULTADOS E DISCUSSÃO}

Durante o período sob refrigeração, observaram-se podridões apenas nos frutos armazenados a $12{ }^{\circ} \mathrm{C}$, por 21 dias ou mais, com níveis de 50,0 $66,7 \%$. Tal ocorrência pode ser atribuída ao efeito das baixas temperaturas $\left(2{ }^{\circ} \mathrm{C}\right.$ e $\left.5^{\circ} \mathrm{C}\right)$ no retardo do amadurecimento, o que contribuiu para a supressão do desenvolvimento de patógenos. Acosta et al. (2000) também detectaram, em mangas 'Haden' armazenadas a $13{ }^{\circ} \mathrm{C}$ por 28 dias, a ocorrência de $78 \%$ de frutos com podridões.

Durante o período de armazenamento refrigerado, observou-se a ocorrência de danos pelo frio ou chilling nos frutos armazenados a $2{ }^{\circ} \mathrm{C}$ ou $5{ }^{\circ} \mathrm{C}$ em 7 dias, os quais foram caracterizados por lesões em $25-50 \%$ da superfície dos frutos, que evoluíram para mais de $50 \%$ desta superfície em 14 ou 21 dias, respectivamente. Tais sintomas foram expressos pelo aparecimento de manchas de coloração marrom, que inicialmente tinham tamanho pequeno, mas que se tornaram enegrecidas e com maior tamanho, e pela escaldadura superficial na casca. Esse resultado indica que os frutos da cv. Palmer são mais sensíveis à injúria pelo frio que os da 'Tommy Atkins' (MIGUEL et al., 2010), o que não foi o relatado por Nunes et al. (2007).

Ao longo do período de refrigeração, os frutos 
armazenados a $2{ }^{\circ} \mathrm{C}$ e a $5{ }^{\circ} \mathrm{C}$ apresentaram diminuição da taxa respiratória de $43,84 \mathrm{mLCO}_{2} \mathrm{~kg}^{-1} \mathrm{~h}^{-1} \mathrm{e}$ $47,39 \mathrm{mLCO}_{2} \mathrm{~kg}^{-1} \mathrm{~h}^{-1}$ para $10,69 \mathrm{mLCO}_{2} \mathrm{~kg}^{-1} \mathrm{~h}^{-1} \mathrm{e}$ $8,17 \mathrm{mLCO}_{2} \mathrm{~kg}^{-1} \mathrm{~h}^{-1}$, em 7 dias, respectivamente, e manteve-se estável. Nos frutos mantidos a $12{ }^{\circ} \mathrm{C}$, essa atividade apresentou redução até o $7^{\circ}$ dia, seguido de aumento durante o período de armazenamento e evolução do amadurecimento (Figura 1). Esses resultados indicam o efeito da refrigeração na redução da atividade metabólica dos frutos (CHITARRA; CHITARRA, 2005).

A perda de massa aumentou durante o período de armazenamento, com os frutos mantidos a $12{ }^{\circ} \mathrm{C}$ apresentando maior perda de massa a partir do $14^{\circ}$ dia, enquanto os armazenados a $2{ }^{\circ} \mathrm{C}$ ou $5^{\circ} \mathrm{C}$ apresentaram as menores perdas percentuais e sem diferença significativa entre si, até o $21^{\circ}$ dia (Tabela 1 ). Nunes et al. (2007) também verificaram perdas de massa, da ordem de $3 \%$, em mangas 'Palmer' armazenadas a $2{ }^{\circ} \mathrm{C}$ ou $5{ }^{\circ} \mathrm{C}$ por 20 dias.

Os frutos armazenados a $12{ }^{\circ} \mathrm{C}$ apresentaram aumento nos valores de luminosidade, ângulo hue e cromaticidade da casca a partir do $21^{\circ}$ dia, indicando que a coloração destes frutos evoluiu de vermelho escuro para amarelo brilhante, o que também foi observado por Nunes et al. (2007). Nos armazenados a $2{ }^{\circ} \mathrm{C}$ e a $5{ }^{\circ} \mathrm{C}$, não se observou variação na luminosidade ou na cromaticidade, mas aumento no ângulo hue, indicando que esta coloração se tornou mais escurecida, o que está relacionado à ocorrência de injúrias pelo frio (Tabela 2), e são indicadores da perda de qualidade (CHITARRA, 1994; O`HARE, 1995).

As condições de armazenamento testadas não interferiram na coloração da polpa, cuja luminosidade $(82,14 \pm 0,66)$, ângulo hue $(101,48 \pm 1,55) \mathrm{e}$ cromaticidade $(34,62 \pm 0,67)$ se mantiveram estáveis, indicando a manutenção da coloração amarela característica, conforme o observado por Morais e Assis (2004) em mangas 'Tommy Atkins'.

A firmeza da polpa aumentou significativamente na primeira semana e manteve-se nos arma- zenados a $2{ }^{\circ} \mathrm{C}$ ou a $5{ }^{\circ} \mathrm{C}$, enquanto nos mantidos a $12{ }^{\circ} \mathrm{C}$ ela se reduziu após o $21^{\circ}$ dia, indicando o amadurecimento normal dos mesmos (Tabela 3 ).

Ao longo do período de armazenamento, verificou-se aumento expressivo no conteúdo de sólidos nos frutos mantidos a $12{ }^{\circ} \mathrm{C}$, enquanto nos armazenados a $2^{\circ} \mathrm{C}$ ou $5^{\circ} \mathrm{C}$, estes teores mantiveram-se estáveis (Tabela 3 ), indicando que os mantidos a $12{ }^{\circ} \mathrm{C}$ amadureceram. $\mathrm{O}$ aumento no teor de sólidos solúveis é parte do processo normal de amadurecimento em mangas, sendo decorrente dos processos de degradação dos polissacarídeos (KNEE; BARTLEY, 1981). Morais e Assis (2004) também relataram que o conteúdo de sólidos solúveis em mangas 'Tommy Atkins', durante o armazenamento refrigerado, apresentou correspondência direta com a temperatura.

Os teores de acidez em frutos armazenados a $12{ }^{\circ} \mathrm{C}$ reduziram-se significativamente a partir do $21^{\circ}$ dia, enquanto nos conservados a $2{ }^{\circ} \mathrm{C}$ ou $5{ }^{\circ} \mathrm{C}$, ela permaneceu constante (Tabela 3 ). $\mathrm{O}$ decréscimo verificado nos frutos armazenados a $12{ }^{\circ} \mathrm{C}$ pode ser atribuído à utilização dos ácidos como substratos respiratórios ou pela conversão em açúcares (BRODY, 1996).

O sabor adocicado dos frutos é devido, em grande parte, ao balanço entre ácidos e açúcares, o qual pode ser estimado pela relação SS/AT, que para o mercado brasileiro, as mais elevadas são as mais desejáveis (THÉ et al., 2001). Neste trabalho, observou-se que esta relação foi influenciada pela temperatura e pelo período de armazenamento, que refletiram diretamente a variação nos teores de sólidos solúveis e acidez titulável (Tabela 3 ).

Nos frutos mantidos a $12{ }^{\circ} \mathrm{C}$, a relação $\mathrm{SS} /$ AT aumentou durante o armazenamento como consequência do aumento nos teores de sólidos solúveis e de redução nos de acidez. Nos frutos armazenados a $2{ }^{\circ} \mathrm{C}$ ou $5{ }^{\circ} \mathrm{C}$, estes valores não variaram, indicando retenção no amadurecimento, o que também foi verificado por Morais e Assis (2004) em mangas 'Tommy Atkins' mantidas a $6^{\circ} \mathrm{C}, 7^{\circ} \mathrm{C}$ ou $8^{\circ} \mathrm{C}$. 


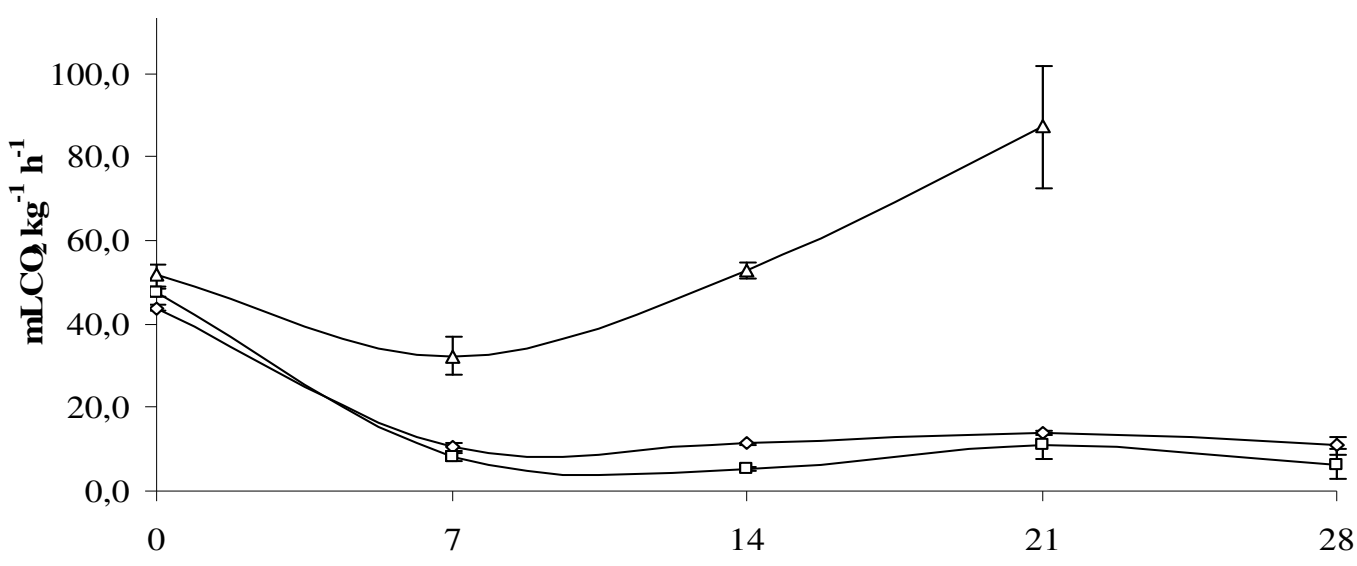

Armazenamento (dia)

$\multimap-2^{\circ} \mathrm{C} \rightarrow-5^{\circ} \mathrm{C} \neg-12^{\circ} \mathrm{C}$

FIGURA 1 - Atividade respiratória em frutos de mangueira 'Palmer' armazenadas a $2{ }^{\circ} \mathrm{C}(75,7 \% \mathrm{UR}), 5$ ${ }^{\circ} \mathrm{C}(73,8 \% \mathrm{UR})$ e $12{ }^{\circ} \mathrm{C}(82 \% \mathrm{UR})$, por até 28 dias. (Barras verticais representam o erro-padrão da média).

TABELA 1 - Valores médios de perda de massa fresca (\%) por mangueiras 'Palmer', armazenadas sob diferentes temperaturas, por até 28 dias.

\begin{tabular}{lccccc}
\hline \multirow{2}{*}{ Temperatura } & \multicolumn{5}{c}{ Tempo de armazenamento (dia) } \\
\cline { 2 - 6 } & $\mathbf{0}$ & $\mathbf{7}$ & $\mathbf{1 4}$ & $\mathbf{2 1}$ & $\mathbf{2 8}$ \\
\hline $\mathbf{2}^{\circ} \mathbf{C}$ & $0,00 \mathrm{eA}$ & $2,23 \mathrm{dA}$ & $3,38 \mathrm{cB}$ & $4,57 \mathrm{bB}$ & $5,76 \mathrm{aC}$ \\
$\mathbf{5}^{\circ} \mathbf{C}$ & $0,00 \mathrm{eA}$ & $2,04 \mathrm{dA}$ & $3,70 \mathrm{cB}$ & $5,32 \mathrm{bB}$ & $6,90 \mathrm{aB}$ \\
$\mathbf{1 2}^{\circ} \mathbf{C}$ & $0,00 \mathrm{eA}$ & $2,50 \mathrm{dA}$ & $4,99 \mathrm{cA}$ & $7,69 \mathrm{bA}$ & $10,82 \mathrm{aA}$ \\
\hline
\end{tabular}

Médias seguidas da mesma letra, minúscula nas linhas e maiúscula nas colunas, não diferem entre si, pelo teste de Tukey $(\mathrm{P}<0,05)$.

TABELA 2 - Coloração da casca de mangueira 'Palmer', armazenadas sob diferentes temperaturas, por até 28 dias.

\begin{tabular}{|c|c|c|c|c|c|}
\hline \multirow{2}{*}{ Temperatura } & \multicolumn{5}{|c|}{ Tempo de armazenamento (dia) } \\
\hline & $\mathbf{0}$ & 7 & 14 & 21 & 28 \\
\hline \multicolumn{6}{|c|}{ Luminosidade } \\
\hline $2^{\circ} \mathrm{C}$ & $38,49 \mathrm{aA}$ & $35,00 \mathrm{aB}$ & $40,65 \mathrm{aA}$ & $39,61 \mathrm{aB}$ & $36,70 \mathrm{aB}$ \\
\hline $5^{\circ} \mathrm{C}$ & $38,48 \mathrm{aA}$ & $42,19 \mathrm{aA}$ & $38,74 \mathrm{aA}$ & $43,57 \mathrm{aB}$ & $38,98 \mathrm{aAB}$ \\
\hline $12{ }^{\circ} \mathrm{C}$ & $38,48 \mathrm{bcA}$ & $36,55 \mathrm{cB}$ & $42,56 \mathrm{bcA}$ & $58,29 \mathrm{aA}$ & $44,01 \mathrm{bA}$ \\
\hline \multicolumn{6}{|c|}{ ângulo hue } \\
\hline $2^{\circ} \mathrm{C}$ & $33,56 \mathrm{cA}$ & $38,31 \mathrm{bcB}$ & $83,48 \mathrm{aA}$ & $81,21 \mathrm{aA}$ & $62,09 \mathrm{abB}$ \\
\hline $5^{\circ} \mathrm{C}$ & $33,56 \mathrm{bA}$ & $71,02 \mathrm{aA}$ & $43,15 \mathrm{bB}$ & $68,37 \mathrm{aA}$ & $90,09 \mathrm{aA}$ \\
\hline $12{ }^{\circ} \mathrm{C}$ & $33,56 \mathrm{cA}$ & $51,52 \mathrm{bcAB}$ & $49,31 \mathrm{bcB}$ & $69,97 \mathrm{abA}$ & $91,19 \mathrm{aA}$ \\
\hline \multicolumn{6}{|c|}{ Cromaticidade } \\
\hline $2^{\circ} \mathrm{C}$ & $12,20 \mathrm{aA}$ & $13,88 \mathrm{aA}$ & $17,95 \mathrm{aA}$ & $16,08 \mathrm{aB}$ & $13,98 \mathrm{aB}$ \\
\hline $5^{\circ} \mathrm{C}$ & $12,20 \mathrm{aA}$ & $18,68 \mathrm{aA}$ & $14,21 \mathrm{aA}$ & $13,05 \mathrm{aB}$ & $15,53 \mathrm{aB}$ \\
\hline $12^{\circ} \mathrm{C}$ & $12,20 \mathrm{cA}$ & $14,14 \mathrm{cA}$ & $15,43 \mathrm{cA}$ & $36,49 \mathrm{aA}$ & $23,24 \mathrm{bA}$ \\
\hline
\end{tabular}

Médias seguidas da mesma letra, minúscula nas linhas e maiúscula nas colunas, não diferem entre si, pelo teste de Tukey ( $\mathrm{P}<0,05)$. 
TABELA 3 - Firmeza, teores de sólidos solúveis (SS), acidez titulável (AT) e relação SS/AT em mangueira 'Palmer', armazenadas sob diferentes temperaturas, por até 28 dias.

\begin{tabular}{|c|c|c|c|c|c|}
\hline \multirow{2}{*}{ Temperatura } & \multicolumn{5}{|c|}{ Tempo de armazenamento (dia) } \\
\hline & $\mathbf{0}$ & 7 & 14 & 21 & 28 \\
\hline \multicolumn{6}{|c|}{ Firmeza (N) } \\
\hline $2^{\circ} \mathrm{C}$ & $65,11 \mathrm{bA}$ & $121,65 \mathrm{aA}$ & $118,46 \mathrm{aA}$ & $127,53 \mathrm{aA}$ & $127,53 \mathrm{aA}$ \\
\hline $5^{\circ} \mathrm{C}$ & $65,11 \mathrm{bA}$ & $123,85 \mathrm{aA}$ & $125,08 \mathrm{aA}$ & $122,38 \mathrm{aA}$ & $127,53 \mathrm{aA}$ \\
\hline $12^{\circ} \mathrm{C}$ & $65,11 \mathrm{bA}$ & $119,44 \mathrm{aA}$ & $121,16 \mathrm{aA}$ & $114,53 \mathrm{aA}$ & $49,19 \mathrm{bB}$ \\
\hline \multicolumn{6}{|c|}{ SS ( $\left({ }^{\circ}\right.$ Brix $)$} \\
\hline $2^{\circ} \mathrm{C}$ & $6,50 \mathrm{aA}$ & $5,97 \mathrm{abB}$ & $5,57 \mathrm{bB}$ & $6,10 \mathrm{abB}$ & $6,00 \mathrm{abB}$ \\
\hline $5^{\circ} \mathrm{C}$ & $6,50 \mathrm{aA}$ & $5,97 \mathrm{abB}$ & $5,77 \mathrm{aB}$ & $6,27 \mathrm{aB}$ & $6,37 \mathrm{aB}$ \\
\hline $12{ }^{\circ} \mathrm{C}$ & $6,50 \mathrm{dA}$ & $8,07 \mathrm{cA}$ & $7,20 \mathrm{dA}$ & $15,87 \mathrm{aA}$ & $14,27 \mathrm{bA}$ \\
\hline \multicolumn{6}{|c|}{ AT (g ác. cítrico $100 \mathrm{~g}^{-1}$ ) } \\
\hline $2^{\circ} \mathrm{C}$ & $1,051 \mathrm{aA}$ & $1,229 \mathrm{aA}$ & $1,308 \mathrm{aA}$ & $1,031 \mathrm{aA}$ & $1,032 \mathrm{aA}$ \\
\hline $5^{\circ} \mathrm{C}$ & $1,051 \mathrm{aA}$ & $1,149 \mathrm{aA}$ & $1,300 \mathrm{aA}$ & $1,218 \mathrm{aA}$ & $1,072 \mathrm{aA}$ \\
\hline $12{ }^{\circ} \mathrm{C}$ & $1,051 \mathrm{aA}$ & $1,128 \mathrm{aA}$ & $1,409 \mathrm{aA}$ & $0,547 \mathrm{bB}$ & $0,443 \mathrm{bB}$ \\
\hline \multicolumn{6}{|c|}{ SS/AT } \\
\hline $2{ }^{\circ} \mathrm{C}$ & $6,19 \mathrm{aA}$ & $4,99 \mathrm{aA}$ & $4,25 \mathrm{aA}$ & $4,96 \mathrm{aB}$ & $5,89 \mathrm{aB}$ \\
\hline $5^{\circ} \mathrm{C}$ & 6,19 aA & $5,23 \mathrm{aA}$ & $4,43 \mathrm{aA}$ & $5,24 \mathrm{aB}$ & $6,61 \mathrm{aB}$ \\
\hline $12^{\circ} \mathrm{C}$ & $6,19 \mathrm{bA}$ & $7,17 \mathrm{bA}$ & $5,21 \mathrm{bA}$ & $30,80 \mathrm{aA}$ & $32,26 \mathrm{aA}$ \\
\hline
\end{tabular}

Médias seguidas da mesma letra, minúscula nas linhas e maiúscula nas colunas, não diferem entre si, pelo teste de Tukey ( $\mathrm{P}<0,05)$.

\section{CONCLUSÕES}

1 - Mangas 'Palmer' armazenadas a $2{ }^{\circ} \mathrm{C}$ ou $5{ }^{\circ} \mathrm{C}$ apresentaram injúrias pelo frio em 7 dias, exteriorizadas pelo escurecimento da casca, sem diferenças quanto à intensidade dos danos. Não se observou prejuízo ao desenvolvimento da coloração da polpa e paralisação no amadurecimento dos frutos, indicado pelo retardo no amolecimento e na solubilização dos açúcares.

2 - Os frutos armazenados a $12{ }^{\circ} \mathrm{C}$ não apresentaram danos pelo frio ou prejuízos ao desenvolvimento do amadurecimento.

\section{AGRADECIMENTOS}

À Fundação de Amparo à Pesquisa do Estado de São Paulo (FAPESP), pelo auxílio financeiro (Proc. $n^{\circ}$ 09/51977-9).

\section{REFERÊNCIAS}

ACOSTA, R.M.; NEITO, A.D.; MENA, N.G.; TELIZ, O.D.; VAQUERA, H.H.; NIETO, A.R. Effect of post-harvest temperatures on the development of internal darkening in mango fruits (Mangifera indica L.) cv. Haden and their quality. Acta Horticulturae, The Hague, v. 509, p. 401-412, 2000.
AOAC. Official methods of analysis. 18. Gaithersburg: AOAC, 2005. chap. 37, p. 10-11.

BRODY, A.L. Envasado de alimentos em atmosferas controladas, modificadas y a vacio. Zaragoza: Acribia, 1996. p. 220.

CHITARRA, M.I.F. Colheita e qualidade pós-colheita de frutos. Informe Agropecuário, Belo Horizonte, v. 17, n. 179, p. 8-18, 1994.

CHITARRA, M.I.F.; CHITARRA, A.B. Pós-colheita de frutas e hortaliças: fisiologia e manuseio. 2.ed. Lavras: UFLA, 2005. 785 p.

FAO. ORGANIZAÇÃO DAS NAÇÕES UNIDAS PARA ALIMENTAÇÃO E AGRICULTURA. Disponível em: $<$ http://www.fas.fao.org $>$. Acesso em: 09 jun. 2011.

KNEE, M.; BARTLEY, I.M. Composition and metabolism of cell wall polysaccharides in ripening fruits. In: FRIED, J.; RHODES, M.J.C. (Ed.). Recent advances in the biochemistry of fruits and vegetables. New York: Academic Press, 1981. p. 131-146. 
LIMA, M.A.C.; SILVA, A.L.; AZEVEDO, S.S.N.; SANTOS, P.S. Tratamentos pós-colheita com 1-metilciclopropeno em manga 'Tommy Atkins': efeito de doses e número de aplicações. Revista Brasileira de Fruticultura, Jaboticabal, v. 28, n. 1, p. 64-68, 2006.

MIGUEL, A.C.A.; DURIGAN, J.F.; MORGADO, C.M.A.; GOMES, R.F.O.; SEIXAS, T.; GUEDES, A.C.T.P. Efeito do dano pelo frio na qualidade de mangas 'Tommy Atkins'. In: CONGRESSO BRASILEIRO DE FRUTICULTURA, 21., 2010, Natal. Frutas: saúde, inovação e sustentabilidade. Natal: RBF, 2010. CD-ROM.

MORAIS, P.L.D. de; ASSIS, J.S. de. Quality and conservation of mango 'Tommy Atkins' as affected by maturity stage and storage temperature. Acta Horticulturae, The Hague, v. 645, p. 639-643, 2004.

MUÑOZ, T.; RUIZ-CABELLO, J.; MOLINAGARCIA, A. D.; ESCRIBANO, M. I.; MERODIO, C. Chilling temperature storage changes the inorganic phosphate pool distribution in cherimoya (Annona cherimola) fruit. Journal of the American Society for Horticultural Science, Alexandria, v. 126, n. 1, p. 122-127, 2001.

NUNES, M.C.N.; EMOND, J.P.; BRECHT, J.K.; DEA, S.; PROULX, E. Quality curves for mango fruit (cv. Tommy Atkins and Palmer) stored at chilling and nonchilling temperatures. Journal of Food Quality, Connecticut, v. 30, p. 104-120, 2007.
O'HARE, T.J. Effect of ripening temperature on quality and compositional changes of mango (Mangifera indica L.) cv. Kensington. Australian Journal of Experimental Agriculture, Victoria, v. 35, p. 259 263, 1995.

PHAKAWATMONGKOL, W.; KETSA, S.; DOORN, W.G. Variation in fruit chilling injury among mango cultivars. Postharvest Biology and Technology, Amsterdam, v. 32, n. 1, p. 115-118, 2004.

THÉ, P.M.P.;CARVALHO, V.D de; ABREU, C.M.P. de; NUNES, R. de P.; PINTO, N.A.V.D. Efeito da temperatura de armazenamento e do estádio de maturação sobre a composição química do abacaxi cv. Smooth Cayenne L. Ciência e Agrotecnologia, Lavras, n. 25, v. 2, p. 356-363, 2001.

WANG, B.; WANG, J.; LIANG, H.; YI, J.; ZHANG, J.; LIN, L.; WU, Y.; FENG, X.; CAO, J.; JIANG, W. Reduced chilling injury in mango fruit by 2,4-dichlorophenoxyacetic acid and the antioxidant response. Postharvest Biology and Technology, Amsterdam, v. 48, n. 2, p. 172-181, 2008.

WHANGCHAI, K.; GEMMA, H.; IWAHORI, S.; UTHAIBUTRA, J. Endogenous polyamines in 'Nam Dok Mai' mangoes with different ripening stages and its relation to chilling injury during storage. Acta Horticulturae, The Hague, v. 509, p. 429-433, 2000. 